\title{
Surface Property and Stability of Transparent Superhydrophobic Coating Based on $\mathrm{SiO}_{2}$-Polyelectrolyte Multilayer
}

\author{
Sunisa JINDASUWAN ${ }^{1}$, On-uma NIMITTRAKOOLCHAI ${ }^{2}$, Sitthisuntorn SUPOTHINA ${ }^{2}$ * \\ ${ }^{1}$ Department of Industrial Chemistry, Faculty of Applied Science, King Mongkut's University of Technology North \\ Bangkok, Bangkok, 10800, Thailand \\ ${ }^{2}$ National Metal and Materials Technology Center, National Science and Technology Development Agency (NSTDA), 114 \\ Thailand Science Park Paholyothin Rd., Klong Luang, Pathumthani, 12120, Thailand
}

ref $\mathrm{http} / / / \mathrm{dx}$. doi.org/10.5755/j01.ms.22.2.12952

Received 22 August 2015; accepted 15 January 2016

\begin{abstract}
Artificial superhydrophobic films were deposited onto a glass slide by performing layer-by-layer deposition of 3.5 bilayers of poly(allylamine hydrochloride)/ poly(acrylic acid) polyelectrolyte, followed by a layer of $\mathrm{SiO}_{2}$ nanoparticles of various amounts to enhance the surface roughness and a fluorosilane to reduce the surface free energy. Higher $\mathrm{SiO}_{2}$ content incorporated into the films resulted in rougher surface and higher water contact angle. The total surface free energy determined by using the Owens-Wendt equation dramatically decreased from $31.46 \mathrm{~mJ} \cdot \mathrm{m}^{-2}$ for the film having the relatively flat surface to only $1.16 \mathrm{~mJ} \cdot \mathrm{m}^{-2}$ for the film having the highest surface roughness of $60.2 \pm 1.1$ $\mathrm{nm}$. All the films were optically transparent and had excellent adhesion based on the peel test. Indoor and accelerated weathering tests revealed good weathering stability.

Keywords: superhydrophobic, water-repellent, polyelectrolyte, biomimetic, lotus effect.
\end{abstract}

\section{INTRODUCTION}

Solid surface can interact with its surrounding that comes into contact with it in many ways. For the surface that is in contact with the liquid, it is known that the minimization of total energy of the system is reflected by wetting ability of the solid surface by the liquid. In case of a superhydrophobic surface, the wetting ability is controlled by two parameters: the surface-free energy and the surface roughness. The degree of hydrophobicity can be increased, and thus the wetting ability decreased, with the decrease of the surface free energy and the increase of the surface roughness. A variety of superhydrophobic surfaces has been fabricated by increasing the surface roughness and reducing the surface free energy by using several fabricating methods [1-9].

It is practically difficult to determine the surface energy between the solid and the liquid directly by an experimental measurement. However, it can be calculated based on the measurement of a contact angle between the solid and the liquid. The contact angle, the angle between the solid surface and the static droplet of the liquid, is determined by a balance between three interfacial surface energies: the liquid/vapor surface energy $\left(\gamma_{L V}\right)$, the solid/vapor surface energy $\left(\gamma_{S V}\right)$ and the solid/liquid surface energy $\left(\gamma_{S L}\right)$. For each interface, each point on the threephase junction is three vectors. An equilibrium relation between these vectors is known as Young's equation [10]. The solid/liquid surface energy has been widely discussed by modification of Young's equation. Owens and Wendt proposed the method of measuring polar and dispersive components of the surface-free energy from the contact angles measured by using polar and non-polar liquids [11]. This approach has been used to measure surface-free

\footnotetext{
* Corresponding author. Tel.: +66-2-5646500; fax: +66-2-5646447.

E-mail address: sitthis@mtec.or.th (S. Supothina)
}

energy of polymer-based coatings such as polymethyl siloxane and octyl triethoxysilane coated onto the $\mathrm{TiO}_{2}$ pigment [12], latex in the kaolin pigment coating [13], and surface-modified multi-walled carbon nanotubes [14].

Recently, the superhydrophobic coating based on $\mathrm{SiO}_{2}$-polymer hybrid material has been widely investigated due to its interesting properties, such as an optical transparency, a specific electrical and mechanical behavior, a thermal and weathering resistance, an abrasion and impact resistance $[2,15]$. In this study, the superhydrophobic $\mathrm{SiO}_{2}$-polyelectrolyte multilayer films were coated onto a glass substrate by layer-by-layer deposition of the positively charged poly(allylamine hydrochloride) (PAH) and the negatively charged poly(acrylic acid) (PAA) polyelectrolytes to obtain 3(PAH/PAA) bilayers and another layer of the PAH, followed by a layer of $\mathrm{SiO}_{2}$ nanoparticles and fluorosilane, respectively. The oppositely charged PAH/PAA polyelectrolytes were selected due to their ionic cross linking in the bilayer thus yielding high durability. The use of the positively charged PAH as the last layer is beneficial for the subsequent deposition of the $\mathrm{SiO}_{2}$ nanoparticles, which are negatively charged. The relationship between the hydrophobicity, the surface roughness and the surfacefree energy of the film was investigated. To alter the film's surface roughness, different amount of the $\mathrm{SiO}_{2}$ nanoparticles was incorporated into the film. To access the possibility for a practical use, the film's weathering stability was evaluated by means of an accelerated weathering test and an indoor exposure.

\section{EXPERIMENTAL DETAILS}

\subsection{Materials}

A poly(allylamine hydrochloride) (PAH, Aldrich) and a poly(acrylic acid) (PAA, Aldrich) having molecular 
weights of 70,000 and 100,000 , respectively, were employed to fabricate the polyelectrolyte bilayers. An Aerosil 200 fumed $\mathrm{SiO}_{2}$ (average particle size $=12 \mathrm{~nm}$, specific surface area $=200 \mathrm{~m}^{2} \cdot \mathrm{g}^{-1}$, JJ-Degussa Chem.) was employed to enhance surface roughness of the coating. A trichloro $(1 \mathrm{H}, 1 \mathrm{H}, 2 \mathrm{H}, 2 \mathrm{H}$-perfluorooctyl) silane (Aldrich) was employed as a surface-energy reducing substance.

\subsection{Film preparation}

A superhydrophobic film was deposited on a $2.5 \mathrm{~cm} \times 5 \mathrm{~cm}$ glass slide, which was cleaned in a sonication bath with ethyl alcohol, followed by acetone and distilled water, respectively. The procedure for film fabrication was the modification of the previous study, which can be explained as follow [16]. Firstly, the cleaned glass slide was coated with 3 bilayers of the PAH/ PAA by alternating deposition of the PAH and PAA by using a dipcoating machine. To obtain strong adhesion with the negatively charged glass slide, the positively charged $\mathrm{PAH}$ layer was deposited as the initial layer by immerging the glass slide into a PAH solution $($ concentration $=0.01 \mathrm{M}$ ) for at least 3 minutes. Then, the specimen was withdrawn at a rate of $0.1 \mathrm{~mm} \mathrm{~s}^{-1}$ followed by rinsing with distilled water. Next, the negatively charged PAA layer was deposited by immerging the PAH-coated specimen into a PAA solution (concentration $=0.01 \mathrm{M}$ ) for at least 3 minutes, withdrawing at the rate of $0.1 \mathrm{~mm} \mathrm{~s}^{-1}$ and rinsing with distilled water. The same procedure repeated two more times, thus 3 PAH/PAA bilayers were obtained. The deposition of another PAH layer was conducted by the same procedure, thus the positively charged 3.5 PAH/PAA bilayers was obtained. Then, the specimen was etched by immerging it in $2.3 \mathrm{M} \mathrm{HCl}$ for 180 minutes, followed by immerging in $1.1 \mathrm{M} \mathrm{HCl}$ for another 180 minutes. The etching was performed to create dimples for the subsequent deposition of $\mathrm{SiO}_{2}$ nanoparticles. After rinsing twice with distilled water, the etched specimen was cured in an oven at $180^{\circ} \mathrm{C}$ for 2 hours. The $\mathrm{SiO}_{2}$ nanoparticles were deposited onto the etched specimen by immerging it in a $0.05,1.0$ or $5.0 \mathrm{wt} . \% \mathrm{SiO}_{2}$ suspension for 5 minutes, and then withdrawing at the rate of $0.01 \mathrm{~mm} \mathrm{~s}^{-1}$. The concentration of $\mathrm{SiO}_{2}$ nanoparticles was varied to alter the surface roughness of the coating. Finally, the specimen was immerged in a trichloro $(1 \mathrm{H}, 1 \mathrm{H}, 2 \mathrm{H}, 2 \mathrm{H}$ perfluorooctyl) silane solution ( 0.4 vol.\% in 1-propanol) followed by curing at $180{ }^{\circ} \mathrm{C}$ for 2 hour. The notations S_0.05, S_1.0 and S_5.0 are referred to specimens fabricated from the $0.05,1.0$ and $5.0 \mathrm{wt} . \% \quad \mathrm{SiO}_{2}$ suspensions, respectively. The film's structure is schematically depicted in the Fig. 1.

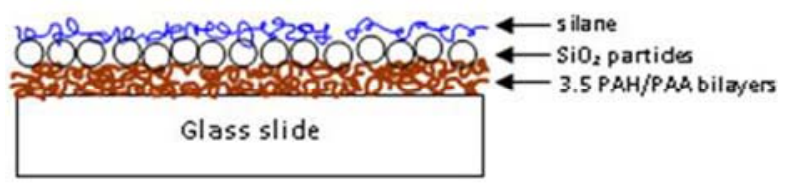

Fig. 1. Schematic illustration of the film's structure

\subsection{Characterization}

An SPA 400 atomic force microscope (AFM, Seiko instruments) was employed for topographical characterization. A non-contact mode was performed using an NSG01 silicon cantilever. A USB4000 UV-visible spectro-photometer (Ocean Optics) was employed for optical transparency measurement. The degree of hydrophobicity was determined by using a goniometer (ramé-hart instrument). The volume of water droplet was $6 \mu \mathrm{L}$. The measurement was conducted for at least 3 times.

The film's weathering stability assessment was performed in a Ci3000 Xenon Weather-Ometer (Atlas Material Testing Solutions) following the ASTM standard (G 155-04a) using a xenon arc light and a water apparatus to simulate a natural weathering condition [17]. Operating parameters for the weathering test are as follow: an irradiation $=0.35 \mathrm{Wm}^{-2} \cdot \mathrm{nm}^{-1}$ at $340 \mathrm{~nm}$; a black panel temperature $=63{ }^{\circ} \mathrm{C}$; a relative humidity $=50 \%$. The testing was conducted for a total time of $500 \mathrm{~h}$ during which the samples were periodically taken out for contactangle measurement. The film's stability was also evaluated by placing the samples at an ambient condition in the laboratory, and measuring the contact angle over a time span of $\sim 4$ weeks.

The adhesion between the film and the glass substrate was evaluated by means of a peel off test following the ASTM standard (D 3359-02) [18]. This test was conducted by applying an adhesive tape (3M Scotch ${ }^{\circledR}$ tape) over cuts made in the film, rubbing with uniform pressure, and then peeling off the adhesive tape. Images of the specimen before and after the tape testing were taken by using an optical microscope to observe the film removed. A classification of $5 \mathrm{~B}, 4 \mathrm{~B}, 3 \mathrm{~B}, 2 \mathrm{~B}, 1 \mathrm{~B}$ and $0 \mathrm{~B}$ was assigned for a percentage of area removal of $0,<5,5-15,16-35$, $36-65$ and $>65 \%$, respectively.

The surface-free energy was calculated from the contact angle data measured by using water and diiodomethane (DIM, 99\%, Aldrich) using the OwensWendt equation as follow [11]:

$1+\cos \theta=\frac{2 \sqrt{\gamma_{S}^{D} \gamma_{L}^{D}}}{\gamma_{L V}}+\frac{2 \sqrt{\gamma_{S}^{P} \gamma_{L}^{P}}}{\gamma_{L V}}$,

and

$\gamma_{S}=\gamma_{S}^{D}+\gamma_{S}^{P}$

where $\theta$ is the contact angle; $\gamma_{S}^{D}$ and $\gamma_{L}{ }^{D}$ are the dispersion component of the solid and the liquid, respectively; $\gamma_{S}{ }^{P}$ and $\gamma_{L}^{P}$ are the polar component of the solid and the liquid, respectively; $\gamma_{L V}$ is the surface-free energy of the liquid; $\gamma_{S}$ is the total surface-free energy of the solid.

Also, based on the Young's equation [10], the surfacefree energy between the solid and the liquid can be calculated by:

$\gamma_{S L}=\gamma_{S V}-\gamma_{L V} \cos \theta$

where $\theta$ is the contact angle; $\gamma_{S L}$ is the surface-free energy between the solid and the liquid; $\gamma_{S V}$ is the surface-free energy between the solid and the vapor; $\gamma_{L V}$ is the surfacefree energy between the liquid and the vapor.

\section{RESULTS AND DISCUSSION}

AFM images taken on the samples containing various amounts of the $\mathrm{SiO}_{2}$ nanoparticles are shown in Fig. 2. The film without $\mathrm{SiO}_{2}$ deposition is given in Fig. $2 \mathrm{a}$ as a 
control sample. This film consists of $3.5 \mathrm{PAH} / \mathrm{PAA}$ bilayers and the silane layer. The surface consists of nanoscaled dimples which were created during acid etching of the 3.5 PAH/PAA bilayers. This sample had the surface roughness of $5.0 \pm 0.25 \mathrm{~nm}$, and the contact angle of $87.0 \pm 0.85 \mathrm{deg}$. It is evident from the images that the amount of the deposited $\mathrm{SiO}_{2}$ nanoparticles increased with an increase of the $\mathrm{SiO}_{2}$ concentration in the suspension. As the $\mathrm{SiO}_{2}$ nanoparticles were incorporated into the film as the surface-roughness enhancer, the films containing the $\mathrm{SiO}_{2}$ nanoparticles possessed much higher surface roughness which were $38.0 \pm 2.5,41.5 \pm 1.2$ and $60.2 \pm 1.1$ $\mathrm{nm}$ for the samples S_0.05, S_1.0 and S_5.0, respectively.
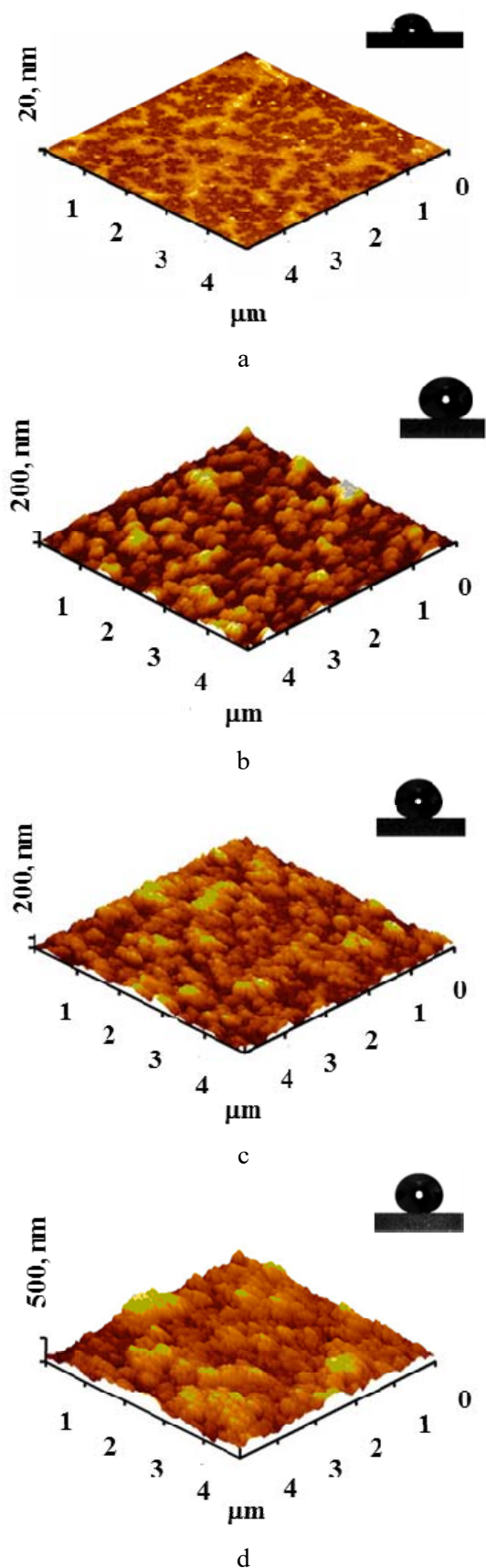

Fig. 2. AFM images of: $\mathrm{a}-$ control sample; $b-\mathrm{S} \_0.05 ; \mathrm{c}-\mathrm{S} \_1.0$; d-S_5.0 samples, respectively
Table 1 summarizes the value of contact angles of water and diiodomethane, and the total surface-free energy. The total surface-free energy calculation took into account both dispersive and polar components at the solid-liquid interface. Rougher surfaces exhibited larger values of the contact angle, which is consistent with the prediction of the Wenzel and Cassie models $[19,20]$. The total surface-free energy of the control sample was $31.46 \mathrm{~mJ} \cdot \mathrm{m}^{-2}$. With the incorporation of the $\mathrm{SiO}_{2}$ nanoparticles as the surfaceroughness enhancer, the total surface-free energy was decreased to $12.54,5.88$ and $1.16 \mathrm{~mJ} \cdot \mathrm{m}^{-2}$ when the surface roughness was increased to $38.0 \pm 2.5,41.5 \pm 1.2$ and $60.2 \pm 1.1 \mathrm{~nm}$, respectively. The plot of the surface roughness and the total surface-free energy versus the contact angle is shown in Fig. 3. To exhibit low wetting ability (i.e., high contact angle), the surface must possess high surface roughness and low surface-free energy $[1,3-5]$.

The result of the film's adhesion testing is as follow. For the sample S_0.05 and S_1.0, there was no removed area observed under the optical microscope after the adhesive tape was peeled off. Thus, the film's adhesion was classified as $5 \mathrm{~B}$, demonstrating a strong adhesion between the film and the glass surface. Flaking along the cuts made in the film of $<5 \%$ was observed on the S_5.0 sample, and thus the adhesion was classified as 4B. Based on the result of the adhesion test, it is evident that the superhydrophobic films prepared under the reported conditions were of sufficient adhesion for the practical use.

Table 1. Values of the contact angle, $\gamma_{S}$ and $\gamma_{S L}$

\begin{tabular}{|c|c|c|c|c|}
\hline Sample & \multicolumn{2}{|c|}{ Contact angle, degree } & \multirow{2}{*}{$\begin{array}{c}\gamma_{S}, \\
\mathrm{~mJ} \cdot \mathrm{m}^{-2}\end{array}$} & $\begin{array}{c}\gamma_{S L}, \\
\mathrm{~mJ} \cdot \mathrm{m}^{-2}\end{array}$ \\
\cline { 2 - 3 } & Water & DIM & 31.46 & 27.68 \\
\hline Control & $87 \pm 0.85$ & $61 \pm 0.31$ & 3.54 & 83.41 \\
\hline S_0.05 & $169 \pm 0.27$ & $99 \pm 0.92$ & 12.54 & 77.19 \\
\hline S_1.0 & $171 \pm 0.69$ & $115 \pm 2.42$ & 5.88 & 72.96 \\
\hline S_5.0 & $174 \pm 0.90$ & $138 \pm 2.16$ & 1.16 & 72 \\
\hline
\end{tabular}

The degree of wetting ability can also be justified by making a comparison of the surface-free energy which is essentially the interfacial energy between the solid surface and the air $\left(\gamma_{S V}\right)$ and the interfacial energy between the solid surface and the liquid $\left(\gamma_{S L}\right)$. Values of the $\gamma_{S L}$ for the samples, which consists of various amount of the $\mathrm{SiO}_{2}$ nanoparticles, are summarized in Table 1 . The $\gamma_{S L}$ of the control sample was $27.68 \mathrm{~mJ} \cdot \mathrm{m}^{-2}$. For the films with higher surface roughness, the $\gamma_{S L}$ increased to a range of $\sim 73-83 \mathrm{~mJ} \cdot \mathrm{m}^{-2}$ which was much higher than the interfacial energy between the film's surface and the air. The increase of the energy at the interface between the film's surface and the liquid means that the system becomes less thermodynamically stable. To be more thermodynamically stable, the solid-liquid interfacial free energy must be minimized. That is, water will bead up on the superhydrophobic surface to reduce this interface area, resulting in high water contact angle. The water droplet can roll off easily on the tilted superhydrophobic surface, and picks up dirt along its path, thus exhibiting selfcleaning property.

The optical transmission spectra of the prepared films at wavelengths ranging from 300 to $700 \mathrm{~nm}$ are shown in Fig. 4. 


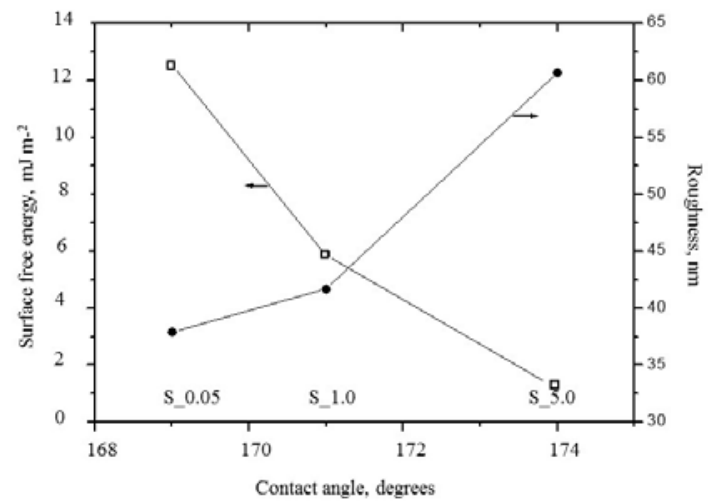

Fig. 3. The plot of the RMS surface roughness and the surfacefree energy versus the contact angle

The S_0.05 and S_1.0 samples had the transmittance of $93 \pm 2 \%$ which was comparable to that of the bare glass substrate indicating their excellent optical transparency. The transmittance of the S_5.0 sample was in a range of $80-90 \%$ which was optically transparent.

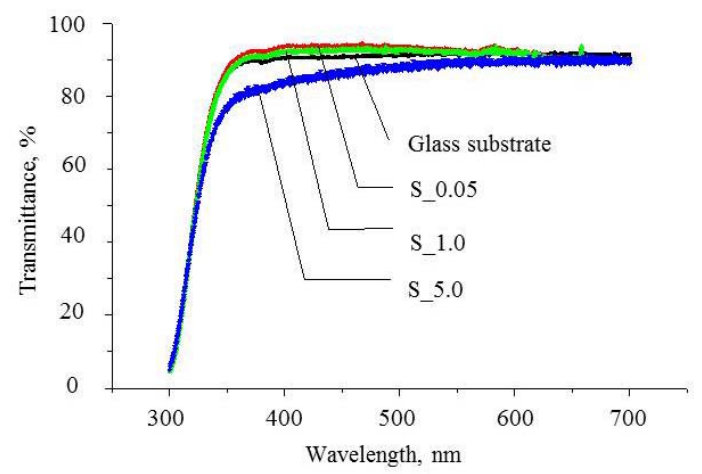

Fig. 4. Transmittance of the uncoated specimen, and the coated specimen consisting various amounts of the $\mathrm{SiO}_{2}$ nanoparticles

Fig. 5 shows the change of the contact angle when the S_5.0 sample was kept in ambient condition in the laboratory for about a month.

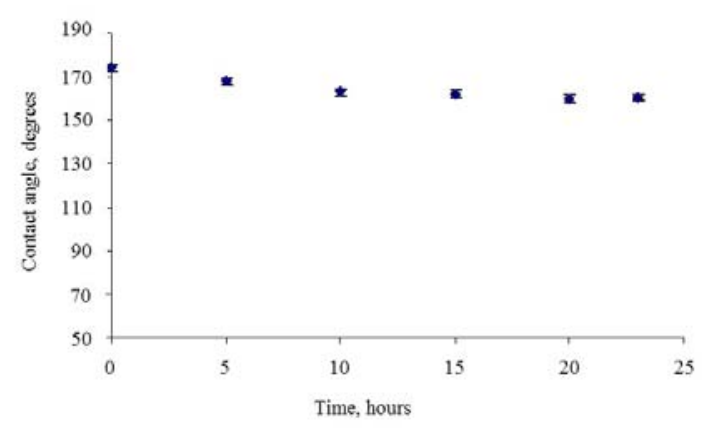

Fig. 5. Indoor weathering stability of the $S \_5.0$ sample

The initial contact angle was $174 \mathrm{deg}$. Upon exposure to ambient atmosphere, the contact angle slightly decreased during the first 10 days because of the accumulation of very fine dust that adhered to the surface, and was constant at $\sim 160 \mathrm{deg}$. afterward. It is known that the hydrophobicity of an artificial superhydrophobic surface gradually degrades over long period of outdoor exposure. For instance, the contact angle of the superhydrophobic silicone nanofilament coating dropped to below $150 \mathrm{deg}$. after 1 week of outdoor exposure [21].

To evaluate its outdoor stability, the S_5.0 sample was subjected to the accelerated weathering test and the result is shown in Fig. 6. Similar to the indoor testing result, the contact angle decreased slightly at an early stage of the testing and became relatively constant after an accelerated weathering exposure for $500 \mathrm{~h}$ which was equivalent to an actual weathering exposure for $\sim 7$ weeks. The result of the accelerated weathering test indicated sufficient stability for the practical use.

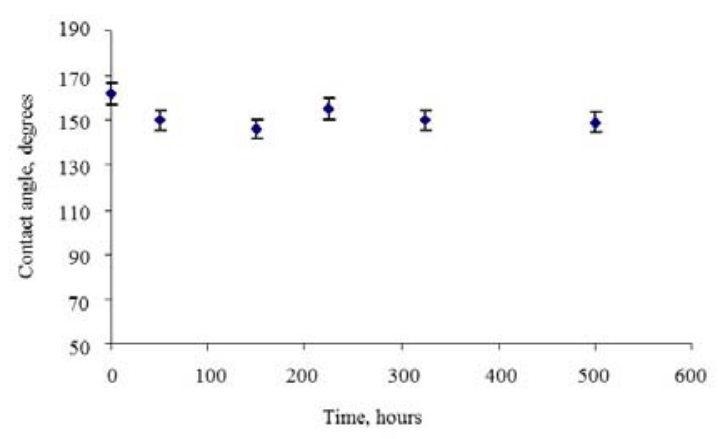

Fig. 6. Accelerated weathering stability of the S_5.0 sample

\section{CONCLUSIONS}

Transparent, superhydrophobic films consisting of 3.5 PAH/PAA polyelectrolyte bilayers, and a layer of the $\mathrm{SiO}_{2}$ nanoparticles and the fluorosilane were coated on glass slides by layer-by-layer deposition. The $\mathrm{SiO}_{2}$ nanoparticles were incorporated into the films at different amount to alter the film's surface roughness. By increasing the $\mathrm{SiO}_{2}$ content, the surface roughness increased resulting in the increase of the water contact angle. The total surface-free energy calculated by the Owens-Wendt equation was in the range of $1.16-12.54 \mathrm{~mJ} \cdot \mathrm{m}^{-2}$. All the prepared films have excellent adhesion based on the peel test, and good stability based on the weathering tests.

\section{Acknowledgement}

The authors would like to acknowledge the financial support of the Nation Metal and Materials Technology Center, NSTDA, Thailand, grant\# MT-B-54-CER-07-277-I).

\section{REFERENCES}

1. Zhai, L., Celeci, F., Cohen, R., Rubner, M. Stable Superhydrophobic Coatings from Polyelectrolyte Multilayers Nano Letters 4 (7) 2004: pp. 1349-1353.

2. Han, J., Zheng, Y., Cho, J., Xurong, X., Cho, K. Stable Superhydrophobic Organic-inorganic Hybrid Films by Electrostatic Self-assembly Journal of Physical Chemistry B 109 (44) 2005: pp. 20773-20778.

3. Shang, H., Wang, Y., Limmer, S., Chou, T., Takahashi, K., Cao, G. Optically Transparent Superhydrophobic Silica-based Films Thin Solid Films $472(1-2)$ 2005: pp. 37-43.

4. Chang, K., Chen, Y., Chen, H. Fabrication of Superhydrophobic Silica-based Surfaces with High 
Transmittance by Using Polypropylene and Tetraethoxysilane Precursors Journal of Applied Polymer Science 107 (3) 2008: pp. 1530 - 1538.

5. Miwa, M., Nakajima, A., Fujishima, A., Hashimoto, K., Watanabe, T. Effects of the Surface Roughness on Sliding Angles of Water Droplets on Superhydrophobic Surfaces Langmuir 16 (13) 2000: pp. 5754-5760.

6. Feng, L., Li, H., Song, Y., Wang, Y. Formation Process of a Strong Water-repellent Alumina Surface by the Sol-gel Method Applied Surface Science 256(10) 2010: pp. $3191-3196$.

7. Ganbavle, V., Bangi, U., Latthe, S., Mahadik, S., Rao, A. Self-cleaning Silica Coatings on Glass by Single Step Solgel Route Surface and Coatings Technology $205(23-24)$ 2011: pp. 5338-5344.

8. Cui, S., Liu, Y., Fan, M., Cooper, A., Lin, B., Liu, X., Han, G., Shen, $\mathbf{X}$. Temperature Dependent Microstructure of MTES Modified Hydrophobic Silica Aerogels Materials Letters 65 (4) 2011: pp.606-609.

http://dx.doi.org/10.1016/j.matlet.2010.11.026

9. Kim, T., Ha, S., Jang, N., Kim, J., Kim, J., Park, J., Lee, D., Lee, J., Kim, S., Kim, J. Simple and Cost-effective Fabrication of Highly Flexible, Transparent Superhydrophobic Films with Hierarchical Surface Design ACS Applied Materials \& Interfaces 7 (9) 2015: pp. $5289-5295$. http://dx.doi.org/10.1021/am5086066

10. Young, T. An Essay on the Cohesion of Fluids Philosophical Transactions of the Royal Society of London 95 1805: pp. $65-87$. http://dx.doi.org/10.1098/rstl.1805.0005

11. Owens, D., Wendt, R. Estimation of the Surface Free Energy of Polymers Journal of Applied Polymer Science 13 (8) 1969: pp. $1741-1747$ http://dx.doi.org/10.1002/app.1969.070130815

12. Lim, B., Thomas, N., Sutherland, I. Surface Energy Measurements of Coated Titanium Dioxide Pigment Progress in Organic Coatings 62 (2) 2008: pp. $123-128$.
13. Järnström, J., Väisänen, M., Lehto, R., Jäsberg, A. Timonen, J., Peltonen, J. Effect of Latex on Surface Structure and Wetting of Pigment Coatings Colloids and Surfaces A 353 (2-3) 2010: pp. 104-116.

14. Cho, S., Ko, K. Surface Free Energy and Superhydrophobic Coating of Multi-walled Carbon Nanotubes by 3:1 TMCS/Toluene Glow Discharge Plasma under Low Pressure Thin Solid Films $518(22)$ 2010: pp. $6619-6623$. http://dx.doi.org/10.1016/j.tsf.2010.03.136

15. Ribeiro, T., Baleizão, C., Farinha, J. Functional Films Silica/Polymer Nanoparticles Materials 7 (5) 2014: pp. $3881-3900$.

16. Nimittrakoolchai, O., Supothina, S., Deposition of Organic-based Superhydrophobic Films for Anti-adhesion and Self-cleaning Applications Journal of the European Ceramic Society 28 (5) 2008: pp. 947-952.

17. ASTM G 155-04a: Standard Practice for Operating Xenon Arc Light Apparatus for Exposure of Non-metallic Materials, ASTM International, West Conshohocken, PA, 2004.

18. ASTM D 3359-02: Standard Test Methods for Measuring Adhesion by Tape Test, ASTM International, West Conshohocken, PA, 2002.

19. Wenzel, R. Surface Roughness and Contact Angle The Journal of Physical and Colloid Chemistry 53 (9) 1949: pp. 1466 - 1467. http://dx.doi.org/10.1021/j150474a015

20. Cassie, A. Contact Angle Discussions of the Faraday Society 3 1948: pp. 11-16. http://dx.doi.org/10.1039/df9480300011

21. Zimmermanna, J., Reifler, F., Schrade, U., Artus, G., Seeger, S. Long Term Environmental Durability of a Superhydrophobic Silicone Nanofilament Coating Colloids and Surfaces A 302 (1-3) 2007: pp. 234-240. 